\title{
Inestabilidad ligamentaria de rodilla en pacientes postoperados de fractura de meseta tibial Schatzker II-VI
}

\author{
Ligamentary instability of the knee in postoperative patients \\ with Schatzker II-VI tibial plateau fracture \\ José de Jesús Ruiz-Ruvalcaba, * Carlos Antonio Cuevas-Mora, ${ }^{\ddagger}$ Zamir Alejandro Zarate-Ayup§ \\ *Médico residente del cuarto año de Traumatología y Ortopedia; ${ }^{\ddagger}$ Médico Traumatólogo y Ortopedista; ${ }^{\S}$ Médico adscrito al Servicio \\ de Traumatología y Ortopedia. Hospital General de Zona Núm. 16, Torreón, Coahuila.
}

\begin{abstract}
Resumen
Introducción: Las fracturas de meseta tibial representan 1\% de todas las fracturas, con una distribución entre hombre y mujer de dos a uno, siendo más frecuente entre los 30 y 50 años de edad. Por lo general se presentan en accidentes de alta energía y se asocian a otras lesiones. Estas fracturas pueden representar un desafío cuando ocurren concomitantemente con alguna lesión ligamentaria. Objetivo: Identificar la prevalencia de inestabilidad ligamentaria de rodilla en pacientes postoperados de fractura de meseta tibial Schatzker II-VI. Material y métodos: Se captaron los pacientes por medio de criterios de inclusión, exclusión y eliminación. Se buscaron los expedientes, se comunicó vía telefónica y se citó al área de consulta externa para su valoración mediante exploración física por medio de las maniobras de Lachman, Lachman reverso, bostezos medial y lateral para evaluar si existía algún tipo de inestabilidad ligamentaria. Estudio de serie de casos y control (nivel de evidencia III). Resultados: Se incluyó un total de 45 pacientes como tamaño de muestra final, se observó que $75 \%$ de los pacientes presentaban datos clínicos de prevalencia de inestabilidad ligamentaria de rodilla. Conclusión: Los pacientes con fractura de meseta tibial Schatzker II-VI postoperados cursan con inestabilidad ligamentaria de rodilla, confirmando así la hipótesis inicial mediante los resultados obtenidos.
\end{abstract}

Palabras clave: Meseta tibial, inestabilidad ligamentaria, fractura, Schatzker.

Nivel de evidencia: III

\begin{abstract}
Introduction: Tibial plateau fractures represent $1 \%$ of all fractures; with a distribution between men and women of two to one, being more frequent between 30 and 50 years of age. They generally occur in high energy accidents and associated with other injuries. These fractures can be challenging when they occur concomitantly with a ligament injury. Objective: To identify the prevalence of knee ligament instability in postoperative patients with a Schatzker II-VI tibial plateau fracture. Material and methods: All patients with inclusion criteria were identified, the physical and electronic files were searched, the patient was contacted by telephone and the outpatient department was summoned for their assessment by means of Physical examination and questioning of the different study variables to assess whether there was any type of ligament instability. Results: A total of 45 patients were included as the final sample size. Where it was observed that $75 \%$ of the patients present clinical data on the prevalence of knee ligament instability. Conclusion: Patients with postoperative schatzker II-VI tibial plateau fracture present with knee ligament instability.
\end{abstract}

Keywords: Tibial plateau, ligament instability, fracture, Schatzker.

Level of evidence: III

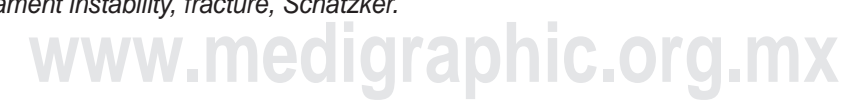

Correspondencia:

Dr. José de Jesús Ruiz Ruvalcaba

Boulevard Revolución s/n esquina Dalias,

Col: Torreón Jardín, 27100, Torreón, Coahuila, Tel: 871 721-2049

E-mail: drjesusruizruv@ hotmail.com
Recibido: 26-02-2021. Aceptado: 28-04-2021

Citar como: Ruiz-Ruvalcaba JJ, Cuevas-Mora CA, Zarate-Ayup ZA. Inestabilidad ligamentaria de rodilla en pacientes postoperados de fractura de meseta tibial Schatzker II-VI. Orthotips. 2021; 17 (3): 143-147. https://dx.doi.org/10.35366/100623 


\section{Introducción}

Las fracturas de meseta tibial representan $1 \%$ de todas las fracturas y hasta $8 \%$ en los pacientes de edad avanzada, con una distribución entre hombre y mujer de dos a uno, siendo más frecuente entre los 30 y 50 años de edad. Se producen tanto por mecanismos directos como indirectos. Por lo general se presentan en accidentes de alta energía y se asocian a otras lesiones de la misma pierna. ${ }^{1}$ Estas fracturas pueden representar un desafío cuando ocurren concomitantemente con alguna lesión ligamentaria. ${ }^{2}$ En asociación a esta patología, puede presentarse la persistencia de inestabilidad ligamentaria como una de sus complicaciones, la cual se describe muy poco en la literatura, pero representa una causa de incapacidad y ausentismo laboral, o con un pronóstico desfavorable para realizar incluso actividades cotidianas, con disminución de la calidad de vida así como aumento de costos institucionales por incapacidad prolongada, incluso llegando a pensionar a algunos pacientes.

Tscherne y Lobenhoffer hicieron énfasis en la importancia de distinguir entre fracturas puras de la meseta tibial y las fracturas-luxaciones. En su revisión de 190 fracturas se produjeron $67 \%$ de lesiones meniscales, $96 \%$ de lesiones de ligamentos cruzados y $85 \%$ de lesiones del ligamento colateral medial. ${ }^{3}$

Si se desprenden en un solo fragmento en forma de cuña o si existe conminación y depresión, a menudo hay afectación de espinas tibiales. ${ }^{4}$

En un estudio realizado en 2005 se evaluaron los daños a 103 pacientes con fractura de meseta tibial mediante resonancia magnética y se observó que sólo $1 \%$ en la serie había completado ausencia de cualquier lesión de tejidos blandos. De los pacientes, 79 (77\%) sufrieron una rotura completa o avulsión de uno o más ligamentos cruzados o lesión de ligamento colateral lateral, 94 pacientes (91\%) mostraron evidencia de lesión meniscal lateral, 45 pacientes (44\%) tenían lesión meniscal medial, 70 pacientes (68\%) presentaron desgarros de una o más de las estructuras que forman la esquina posterolateral. El patrón de fractura más frecuente fue una depresión dividida en meseta lateral $(60 \%)$.

El examen físico de la rodilla y la pierna es crucial para diagnosticar lesiones y complicaciones asociadas con el fin de planificar el tratamiento quirúrgico y decidir el momento óptimo de la intervención. ${ }^{6}$

Aunque muchas veces es complicada la valoración de la integridad de los ligamentos de la rodilla por el dolor que presenta el paciente, ésta puede realizarse bajo anestesia al momento de llevar a cabo la cirugía, lo cual aumenta su sensibilidad; en el caso del ligamento cruzado anterior se puede hacer la prueba de cajón anterior con una sensibilidad de 73\% y especificidad de $95 \%$ cuando se efectúa con anestesia, de igual manera la prueba de Lachman, la cual presenta una sensibilidad y especificidad de 92 y $84 \%$ respectivamente cuando se realiza bajo anestesia.,

La valoración del ligamento cruzado posterior efectuada con la maniobra de Lachman reverso tiene una sensibilidad $62 \%$ y especificidad de $89 \%$, mientras que la maniobra de cajón posterior presenta una sensibilidad y especificidad de 90 y $95 \%$ respectivamente. . $^{3,8}$

La valoración de la integridad de los estabilizadores lateral y mediales como el ligamento colateral lateral y colateral medial se realiza mediante el varus o valgus stress test con sensibilidad de 25 y $85 \%$, respectivamente. ${ }^{3,9}$

En un estudio efectuado en 2017 en 103 pacientes donde se compararon los hallazgos tomográficos con los hallazgos artroscópicos, se encontró que la evaluación de la tomografía preoperatoria puede ayudar a predecir lesiones intraarticulares de tejidos blandos en pacientes con fractura de meseta tibial; un aumento de la depresión de la meseta tibial lateral con un umbral de $11 \mathrm{~mm}$ se asocia con mayor riesgo de desgarro meniscal y mayor incidencia de fractura por avulsión del ligamento cruzado anterior. ${ }^{10}$

Se recomienda resonancia magnética como un estudio auxiliar que se emplea para diagnósticos diferenciales de lesiones de partes blandas en la rodilla con antecedente traumático como ruptura de ligamento cruzado anterior o posterior, luxación, lesiones meniscales o ligamentos colaterales. ${ }^{11}$

No todas las fracturas de la superficie articular tibial proximal requieren cirugía y no todas las fracturas intraarticulares desplazadas necesitan ser reducidas quirúrgicamente, la superficie articular tibial proximal tolera desplazamientos articulares pequeños a moderados, y en fracturas seleccionadas los resultados del tratamiento no quirúrgico presentan excelentes resultados a pesar de las irregularidades articulares. ${ }^{12,13}$

La Sociedad Americana de Cirujanos Ortopédicos establece que para el manejo quirúrgico los pacientes deben tener las siguientes características:

1. El rango de depresión articular no debe rebasar los $10 \mathrm{~mm}$.

2. La inestabilidad de más de $10^{\circ}$ con la rodilla extendida y comparada con la pierna contralateral es indicativo de manejo quirúrgico. 
3. En fracturas abiertas, las cuales van a requerir irrigación y desbridamiento, se recomienda la fijación temporal con fijador externo o realizar de inmediato la fijación interna, pero siempre evitando abrir la articulación.

4. En caso de que se prevea que la fijación interna va a demorar, la fijación externa está indicada cuando la pierna muestra acortamiento o la articulación se encuentra subluxada. ${ }^{14,15}$

Hipótesis: se buscó identificar la prevalencia de inestabilidad ligamentaria de rodilla en pacientes postoperados de fractura de meseta tibial Schatzker II-VI en el periodo de enero de 2018 a diciembre de 2019.

\section{Material y métodos}

Se captaron los pacientes por medio de criterios de inclusión, exclusión y eliminación. Se buscaron los expedientes, se comunicó vía telefónica y se citó al área de consulta externa para su valoración mediante exploración física por medio de las maniobras de Lachman, Lachman reverso, bostezos medial y lateral para evaluar si existía algún tipo de inestabilidad ligamentaria. Estudio de serie de casos y control (nivel de evidencia III) de tipo descriptivo, transversal, observacional y ambispectivo.

\section{Criterios de inclusión}

1. Pacientes derechohabientes del IMSS (Instituto Mexicano del Seguro Social).

2. Edad de 18 años o más con diagnóstico de fractura de meseta tibial Schatzker II-VI con manejo quirúrgico.

3. Ambos sexos.

\section{Criterios de exclusión}

1. Fuera del rango de edad.

2. Manejados de forma conservadora.

3. Expediente clínico incompleto.

4. Pacientes que no desearon participar en el estudio.

5. Pacientes que previo a la fractura contaban con diagnóstico de alguna lesión ligamentaria tanto de la rodilla afectada por la fractura como la rodilla sana.

6. Pacientes que hayan cursado con infección del sitio quirúrgico.

7. Pacientes con retardo en la consolidación o pseudoartrosis.

\section{Criterios de eliminación}

1. Pacientes que no desearon participar en el estudio o que retiraron su consentimiento.

2. No acudieron a su valoración.

Periodo de tiempo: se llevó a cabo de enero de 2018 a diciembre de 2019.

Población elegible a universo: pacientes de 18 años o más que cursaron con fractura de meseta tibial Schatzker II-VI sometidos a algún tipo de manejo quirúrgico.

El presente trabajo se llevó a cabo mediante el uso de estadística descriptiva. Posterior a la captura de datos e identificando los posibles sesgos se realizó un análisis completo, se utilizó el paquete estadístico SPSS 22 para finalmente mostrar los resultados obtenidos en esta investigación.

Se realizó un estudio de casos y controles, el cual es un estudio epidemiológico, observacional y analítico en el que los sujetos se seleccionan en función de que tengan (casos) o no tengan (control) una determinada enfermedad, o en general un determinado efecto.

\section{Resultados}

Con base en el tamaño de muestra considerado, y mediante los criterios de inclusión y exclusión del protocolo, se incluyeron finalmente 45 pacientes, se hizo la captura de variables del expediente clínico sobre las notas registradas en el seguimiento del paciente por el servicio de traumatología. Según los datos obtenidos del presente estudio bajo los objetivos propuestos utilizados para el análisis de variables, se encontraron los siguientes resultados:

De los pacientes analizados se determinó presentar la descripción de las variables en la muestra general $(n=45)$.

Dentro de las características generales de nuestra población y de la descripción de las variables se identificó a los pacientes por grupo de edad, presentándose principalmente en pacientes entre 41 y 65 años de edad.

Para el análisis de la población en relación a la división de los pacientes en cuanto al género, determinamos que $68.88 \%$ corresponde al género masculino. 
En la caracterización de la fractura en cuanto a su tipo según la clasificación de Schatzker, se observó en su mayoría el tipo VI con $27 \%$, la cual corresponde a un trazo articular con afectación de uno o ambos platillos tibiales y disociación de la metáfisis con la diáfisis por presentar un trazo a este nivel.

Dentro de la valoración que se realizó a los pacientes en el presente protocolo, se utilizaron maniobras de exploración física para observar si existe inestabilidad ligamentaria; uno de los signos valorados fue el signo de Lachman, el cual valora el ligamento cruzado anterior.

De la misma forma, se exploró mediante la maniobra de Lachman reverso la evidencia de lesión del ligamento cruzado posterior, no encontrando evidencia de lesión en ninguno de nuestros pacientes de la muestra.

Para la valoración del ligamento colateral lateral se utilizó la maniobra de bostezo lateral o varus stress test; la fractura tipo VI de la clasificación de Schatzker fue la que mayor predominio de lesión del ligamento colateral lateral presentó por clínica, siendo positiva en siete de los 12 pacientes con este tipo de fractura.

La valoración del ligamento colateral medial se realizó con la maniobra de bostezo medial o valgus stress test; la fractura tipo IV de la clasificación de Schatzker fue la que mayor predominio de lesión del ligamento colateral medial presentó por clínica, siendo positiva en cinco de los nueve pacientes con este tipo de fractura.

Dentro de la valoración se detectaron 15 pacientes que mostraron inestabilidad en más de un ligamento al momento de realizar las maniobras de exploración física de la rodilla afectada, siendo la fractura tipo VI de la clasificación de Schatzker la de mayor prevalencia de inestabilidad multiligamentaria.

\section{Discusión}

El presente estudio pretendía demostrar que existe una alta prevalencia de inestabilidad ligamentaria de rodilla en pacientes postoperados de fractura de meseta tibial Schatzker II-VI, por lo que se planeó el estudio con una población de 45 pacientes, los cuales fueron sometidos a manejo quirúrgico en el periodo de enero de 2018 a diciembre de 2019, encontrando que $75 \%$ de los pacientes presentaron datos clínicos de inestabilidad ligamentaria, por lo que los resultados concuerdan con lo descrito en la literatura, donde se reporta la asociación de fractura de meseta tibial y lesión de ligamentos y meniscos hasta en $90 \%$ de los pacientes.

La evidencia que este estudio reveló nos lleva a replantear la manera en la que se valoran los pacientes que presentan una fractura de meseta tibial en nuestro hospital, debido a que $75 \%$ muestran datos de inestabilidad ligamentaria de la rodilla afectada, aun pasado más de un año de la fractura y del evento quirúrgico.

Se confirmó lo que menciona la literatura en cuanto al predominio de este tipo de fracturas en el sexo masculino, el cual se reporta de 2:1 con respecto al sexo femenino; en nuestros resultados $68 \%$ pertenecen al sexo masculino y $32 \%$ al sexo femenino.

El rango de edad que más se ve afectado por esta patología es el de pacientes entre 41-65 años representando $66 \%$, seguido del grupo de edad de $18-40$ años con $31 \%$ y solamente $3 \%$ en el grupo de pacientes mayores de 65 años.

La fractura de meseta tibial tipo VI de la clasificación de Shatzker se reporta como el tipo de trazo de fractura que más predomina con $26 \%$.

Dentro de los signos que valoran inestabilidad ligamentaria de rodilla, el signo de Lachman fue el que más se observó en el grupo de estudio con $35.5 \%$, seguido del bostezo lateral con $24.5 \%$ y bostezo medial con $20 \%$, tomando en cuenta que un porcentaje de pacientes presentó lesión de más de un ligamento; sin embargo, $20 \%$ de los pacientes estudiados no mostraron ningún tipo de evidencia de lesión ligamentaria.

Realizar maniobras de inestabilidad (Lachman, Lachman reverso, bostezo medial y lateral) bajo anestesia al momento de efectuar la cirugía nos da un panorama general del problema al que nos enfrentamos, y aunque la mayoría de las ocasiones es imposible hacer la reparación de un ligamento en agudo, detectar una lesión en éste nos puede orientar hacia cómo llevar el seguimiento y rehabilitación de estos pacientes para valorar si en un futuro es necesario realizar un segundo evento quirúrgico con la finalidad de llevar a cabo algún tipo de plastia ligamentaria.

Dentro de las limitaciones del estudio se encuentran los falsos negativos de las maniobras de exploración cuando se realizan sin anestesia, ya que muchas veces el paciente no se presta a una adecuada exploración por contractura muscular 0 dolor, así como la dificultad de efectuar la reparación ligamentaria en agudo y la rigidez que puede llegar a presentar como secuela de la misma lesión. 


\section{Conclusiones}

Dentro del objetivo del presente estudio se buscó demostrar la prevalencia de inestabilidad ligamentaria de rodilla en pacientes postoperados de fractura de meseta tibial Shatzker II-VI, lo cual confirmó la hipótesis previamente planteada mediante los resultados obtenidos, ya que se evidenció que $75 \%$ de nuestros pacientes mostraron datos de inestabilidad ligamentaria de rodilla a partir de las maniobras de exploración específicas para cada ligamento (LCA, LCP, LCM, $\mathrm{LCL}$ ) en la valoración postquirúrgica.

\section{Referencias}

1. Zhao R, Lin Z, Long H, Zeng M, Cheng L, Zhu Y. Diagnosis and treatment of hyperextension bicondylar tibial plateau fractures. J Orthop Surg Res. 2019; 14 (1): doi: 10.1186/s13018-0191220-z.

2. Cinque ME, Godin JA, Moatshe G, Chahla J, Kruckeberg $\mathrm{BM}$, Pogorzelski J, et al. Do tibial plateau fractures worsen outcomes of knee ligament injuries? a matched cohort analysis. Orthop J Sports Med. 2017; 5 (8): 232596711772389. doi: $10.1177 / 2325967117723895$

3. Norris R, Kopkow C, McNicholas MJ. Interpretations of the dial test should be reconsidered. A diagnostic accuracy study reporting sensitivity, specificity, predictive values and likelihood ratios. Journal of ISAKOS: Joint Disorders \& Orthopaedic Sports Medicine. 2018; 3: 198-204. doi: 10.1136/ jisakos-2018-000197.

4. Freeman MAR, Pinskerova V. The movement of the normal tibio-femoral joint. J Biomech. 2005; 38 (2): 197-208. doi: 10.1016/j.jbiomech.2004.02.006.

5. Gardner MJ, Yacoubian S, Geller D, Suk M, Mintz D, Potter $\mathrm{H}$, et al. The incidence of soft tissue injury in operative tibial plateau fractures. J Orthop Trauma. 2005; 19 (2): 79-84. doi: 10.1097/00005131-200502000-00002.

6. Kfuri M, Schatzker J. Revisiting the Schatzker classification of tibial plateau fractures. Injury. 2018; 49 (12): 2252-2263. doi: 10.1016/j.injury.2018.11.010.

7. Spiro AS, Regier M, Novo de Oliveira A, Vettorazzi E, Hoffmann $\mathrm{M}$, Petersen JP, et al. The degree of articular depression as a predictor of soft-tissue injuries in tibial plateau fracture. Knee Surg Sports Traumatol Arthrosc. 2012; 21 (3): 564-570. doi: 10.1007/s00167-012-2201-5

8. Rubinstein RA, Shelbourne KD, McCarroll JR, VanMeter CD, Rettig AC. The accuracy of the clinical examination in the setting of posterior cruciate ligament injuries. Am J Sports Med. 1994; 22 (4): 550-557. doi: 10.1177/036354659402200419.

9. Molenaars RJ, Mellema JJ, Doornberg JN, Kloen P. Tibial plateau fracture characteristics: computed tomography mapping of lateral, medial, and bicondylar fractures. J Bone Joint Surg Am. 2015; 97 (18): 1512-1520. doi: 10.2106/ jbjs.n.00866.

10. Tang HC, Chen IJ, Yeh YC, Weng CJ, Chang SS, Chen ACY, et al. Correlation of parameters on preoperative CT images with intra-articular soft-tissue injuries in acute tibial plateau fractures: a review of 132 patients receiving ARIF. Injury. 2017; 48 (3): 745-750. doi: 10.1016/j. injury.2017.01.043.

11. Devitt BM, Whelan DB. Physical examination and imaging of the lateral collateral ligament and posterolateral corner of the knee. Sports Med Arthrosc Rev. 2015; 23 (1): 10-16. doi: 10.1097/jsa.0000000000000046.

12. Trasolini NA, Lindsay A, Gipsman A, Rick HGF. The biomechanics of multiligament knee injuries. Clin Sports Med. 2019; 38 (2): 215-234. doi: 10.1016/j.csm.2018.11.009.

13. McGonagle L, Cordier T, Link BC, Rickman MS, Solomon LB. Tibia plateau fracture mapping and its influence on fracture fixation. J Orthop Traumatol. 2019; 20 (1): doi: 10.1186/s10195019-0519-1.

14. Shepherd L, Abdollahi K, Lee J, Vangsness CT. The prevalence of soft tissue injuries in nonoperative tibial plateau fractures as determined by magnetic resonance imaging. J Orthop Trauma. 2002; 16 (9): 628-631. doi: 10.1097/00005131-200210000-00003.

15. Gardner MJ, Yacoubian S, Geller D, Suk M, Mintz D, Potter $\mathrm{H}$, Lorich DG. The incidence of soft tissue injury in operative tibial plateau fractures. J Orthop Trauma. 2005; 19 (2): 79-84. doi: 10.1097/00005131-200502000-00002.

\section{Conflicto de intereses}

Aspectos éticos. El estudio se realizó de acuerdo con lo estipulado en el Reglamento de la Ley General de Salud en Materia de Investigación para la Salud. 